\title{
Genitourinary Applications of Dual-Energy CT
}

\author{
Terri J. Vrtiska ${ }^{1}$, Naoki Takahashi, Joel G. Fletcher, Robert P. Hartman, Lifeng Yu, and \\ Akira Kawashima \\ Department of Radiology, Mayo Clinic, 200 First St., SW, Rochester, MN 55905
}

\begin{abstract}
OBJECTIVE-Recent advances in CT technology provide improved diagnostic characterization of materials using dual-energy CT methods. Application of these methods improves lesion conspicuity and detection. In addition, improved material characterization and creation of virtual unenhanced techniques potentially result in decreased radiation dose. We will review the role of dual-energy CT as applied to the genitourinary system.
\end{abstract}

CONCLUSION-Dual-energy CT is beginning to play an important role in patients with genitourinary diseases by providing unique characterization tools for calculi and masses.

\section{Keywords}

adrenal; calculi; CT; CT urography; dual-energy CT; renal mass; virtual imaging

During the past several years, advances in CT technology have allowed expansion of the armamentarium of diagnostic tools to identify, characterize, and differentiate materials. One of the most promising techniques is the development of dual-energy $\mathrm{CT}$ acquisitions. One of the dual-energy CT techniques is characterized by acquisition of CT data from two different energy spectra, which is referred to as "dual-source, dual-energy CT." This article focuses on the advantages and application of dual-energy CT to the genitourinary system.

\section{Background}

$\mathrm{CT}$ is based on the measurement of the linear attenuation coefficient of each structure at a particular tube potential (or kV). Traditionally, CT acquisitions have been accomplished by acquiring CT data using single tube potentials. Because CT numbers (converted from the linear attenuation coefficient and expressed in Hounsfield units) reflect a combination of the mass attenuation coefficient and the mass density of the material at a given x-ray tube potential, two different materials, such as calcium and iodine, can have the same CT number at a given tube potential. By using two different $\mathrm{x}$-ray spectra, the separation of these materials will be accomplished because each has a unique energy-dependent attenuation for each of the two kVs obtained during one $\mathrm{CT}$ acquisition. From a practical perspective, dualenergy CT must also provide routine anatomic images similar to single-energy CT for diagnostic interpretation. Thus, most dual-energy CT applications will generate both

\footnotetext{
(c) American Roentgen Ray Society

${ }^{1}$ Address correspondence to T. J. Vrtiska (vrtiska.terri@mayo.edu).
} 
material-specific images, which highlight the presence or absence of a specific material, and non-material-specific images, which resemble the images of traditional 120- or 140-kV single-energy CT images.

\section{Hardware Platforms}

Dual-energy CT data can be obtained from several hardware platforms including dualsource CT scanning, rapid kV switching, and multilayer "sandwich" detectors [1] (Table 1). Dual-source CT scanning is acquired with two x-ray tubes and two detectors, with each tube operating at a different tube potential [2]. Non-material-specific images are routinely produced [3], and several dual-source, dual-energy CT material-specific applications for abdominal imaging have already been validated [4-7]. This approach has the advantage of permitting the insertion of different filters for each x-ray tube to widen the spectral separation and large dynamic ranges for tube current for the low-energy spectra, which reduce image noise and should improve material classification. The degree of successful material discrimination generally improves with the degree of spectral separation [8].

Rapid $\mathrm{kV}$ switching is performed using a single $\mathrm{x}$-ray tube system. This approach examines spectral data in projection space and has the advantage of creating monochromatic images and suppressing image artifacts.

The multilayer sandwich $\mathrm{x}$-ray detector is created by the surface layers of the detector measuring low-energy photons and the deeper layers measuring high-energy photons $[1,9]$.

\section{Benefits of Dual-Energy CT}

The clinical use of dual-energy CT applications depends on the unique additional benefits that dual-energy CT provide in comparison with single-energy CT $[1,10]$. Dual-energy CT can benefit patients in several specific areas including improved lesion conspicuity and characterization combined with reduced additional imaging requirements [7]. For example, improved lesion conspicuity is a result of the contribution of the lower tube energy toward highlighting attenuation differences associated with some neoplasms due to differential iodine presence [11], because the iodine signal is substantially increased at $80 \mathrm{kV}$ compared with $120 \mathrm{kV}$ [12]. In general, the images from low-kV scans have not only higher contrast resolution but also higher noise level, whereas images from high-kV scans have lower contrast resolution and lower noise level. Although low-kV data offer dramatically increased contrast-to-noise ratio for small patients, the contribution of the higher-energy data becomes progressively important as patient size increases to maintain image quality. Therefore, dualenergy CT technology is unlikely to be of equal benefit in the abdominal imaging of obese patients when compared with average-sized or small patients because artifacts created by large body habitus at low-energy spectra degrade image quality and therefore prohibit accurate material separation.

Improved lesion characterization is possible using dual-energy CT and material decomposition algorithms where the amount of iodine signal contained in each pixel of the $\mathrm{CT}$ image can be quantified. This signal can be displayed using color over gray-scale images (iodine overlay) or can be subtracted from the gray-scale images (virtual noncontrast). 
Lesion characterization has recently been applied most vigorously to the classification of renal stone types using color-coded visual display [6] and may also be useful in the characterization of renal and adrenal masses $[1,13]$. A reduction in the need for additional imaging is possible by applying lesion characterization algorithms to current CT protocols. For example, by inclusion of virtual noncontrast images in CT urography or renal mass protocols, the need for unenhanced CT studies may become redundant because virtual noncontrast image data sets can be created from the iodine-enhanced images by applying dual-energy CT algorithms. Virtual noncontrast images can also detect urinary stone and renal artery calcification after removing iodinated contrast material from contrast-enhanced scans.

\section{Dual-Energy CT Display}

The display of both non-material-specific and material-specific dual-energy CT images continues to evolve, but there are several alternatives with which abdominal radiologists should be familiar. Non-material-specific images are generally created to display routine anatomic information and can be created to highlight contrast differences and minimize noise or to reflect the CT numbers of single-energy $120-\mathrm{kV}$ data.

Linear blending of high- and low-kV images weights all of the pixels in an image by a fixed factor relative to the tube energy from which they are derived; for example, a 0.3 linear blend derives $30 \%$ of the CT number from the low-energy spectra and 70\% from the highenergy spectra [3]. This mixing is implemented automatically after reconstruction of the images corresponding to each tube energy.

The other method of blending two image sets is nonlinear sigmoidal blending, which maximizes contrast and minimizes noise. This method weights the $\mathrm{CT}$ intensity assigned to individual pixels by their CT number. Pixels with higher $\mathrm{HU}$ values have a higher mixing ratio of low-kV data, whereas pixels with lower $\mathrm{HU}$ values have a lower mixing ratio of low-kV data. This allows greater enhancement of iodine signal than the linear blending method while maintaining low noise level in the low-HU-value regions [3] (Fig. 1). Iodine signal enhancement is particularly useful when only a small amount of iodinated contrast material can be used because of renal insufficiency or only a slow rate of IV injection of iodinated contrast medium is allowed. In addition, decreased urinary excretion of contrast material from an obstruction or diminished renal function may be a good indication for iodine signal enhancement.

Monochromatic images are another flexible way to control image contrast and noise and simulate the CT image of a specific energy. These images can be used to optimize contrastto-noise ratio and obtain quantitative information to characterize materials (by looking at attenuation over a range of $\mathrm{kVs}$ ) [14].

Material-specific images are created to highlight the presence or absence of a material (e.g., virtual noncontrast images are created to display what an image would look like without iodine) [15]. Material classification can be implemented using basic material decomposition in image or projection space. Iodine classification is one of the most useful applications in genitourinary CT and produces both iodine-specific images, which will highlight the 
appearance of subtly enhancing masses, and iodine-subtraction images, which subtract iodine and potentially reveal calcifications or stones that are obscured by the surrounding iodine solution. Iodine-specific images can be displayed by overlaying anatomic images with a color overlay that displays iodine.

Recent improvements in iodine classification permit the measurement of iodinated concentration, but these techniques have not been rigorously tested under a wide variety of clinical conditions. The ability to measure iodine concentration in renal masses may have substantial advantages over traditional CT number measurements, which vary by scanner, reconstruction technique, patient size, and x-ray tube potential [16-18]. Virtual noncontrast images resemble unenhanced images, but generally use noise-reduction methods resulting in some smoothing of the image. Classification of noniodine material but clinically meaningful material is generally performed using color overlay such as in renal stone classification.

Contrary to conventional wisdom, dual-energy CT does not require an increased radiation dose compared with single-energy CT with certain patient size thresholds. Yu et al. [19] recently compared image quality between dose-matched dual-energy CT and single-energy CT in phantoms simulating patients of a variety of sizes. They concluded that

...for adult CT practices, which primarily use $120 \mathrm{kV}$ scanning, the use of dualenergy $\mathrm{CT}$ for the purpose of material-specific imaging can also produce a set of non-material-specific images for routine diagnostic interpretation that are of similar or improved image quality compared to single-energy $120 \mathrm{kV}$ scans.

\section{Applications}

\section{Stone Characterization}

Over the past decade, unenhanced CT has become the method of choice for imaging symptomatic urinary calculi because of its high diagnostic accuracy. In the mid-1990s, studies reported $97 \%$ accuracy for diagnosing ureteral stones $[20,21]$ and subsequent studies reported sensitivities and specificities approaching 100\% [22-25]. However, a shortcoming of CT has been that virtually all urinary calculi (> 99\%) appear as opaque densities on unenhanced CT regardless of composition. Early in vitro studies using single-energy CT attempted to characterize stones using ranges of HUs [26-30]; however, because of overlap in these ranges, it was not possible to apply single-energy spectra data to accurately characterize stones. More recent research including in vitro studies [4, 6, 31-34] and in vivo studies $[5,31,35]$ has used the advantages of dual-energy $\mathrm{CT}$ and the differences in the $\mathrm{x}$ ray attenuation properties at high and low $\mathrm{kVs}$ to allow accurate renal stone differentiation between uric acid (UA)-containing and calcium-containing urinary calculi. The in vitro studies showed a sensitivity of $88-100 \%$ depending on the size of the stone and the size of the anthropomorphic phantom and accuracy of $93-100 \%$. The in vivo data reported sensitivities of 74-100\% and accuracies of $89-100 \%$ including dual-energy CT acquisitions using a low-dose protocol $[5,31,35]$.

These initial studies were directed at differentiation between UA stones versus non-UA stones, with some studies providing visual cues about the stone material using color overlay (Figs. 2 and 3). This binary model provided foundational data to direct clinical care toward 
medical management based on stone type. More recent studies have expanded the characterization tools into a "rainbow" of four or five groups of renal stones including the following: group 1, stones composed of UA, UA dihydrate, and ammonium acid urate; group 2, cystine and struvite; group 3, calcium oxalate monohydrate, dihydrate, and brushite; and group 4, hydroxyapatite, carbonate apatite. Further refinement divides group 2 into two distinct categories for a total of five groups. Stone characterization provides an opportunity to immediately communicate the stone subtype and help direct care for patients with stone disease after dual-energy CT (Qu M, et al., presented at the 2009 annual meeting of the Radiological Society of North America [RSNA]).

\section{Renal Mass}

Renal mass evaluation is a main element of genitourinary CT. The differentiation of a benign cyst from a solid mass is an integral part of any renal CT protocol. Current practice relies heavily on the detection of enhancement within a renal lesion after contrast administration to diagnose a solid renal mass. For this reason, standard renal mass $\mathrm{CT}$ protocols require an unenhanced CT acquisition to establish the baseline attenuation of a renal lesion. A second contrast-enhanced CT acquisition is then performed, and the attenuation of the mass is compared with the baseline unenhanced acquisition. In general, an increase in attenuation of more than $20 \mathrm{HU}$ on contrast-enhanced CT is used as the threshold to identify a solid enhancing mass $[36,37]$.

The ability of dual-energy CT to identify the presence of iodine in body tissues has opened the possibility of single-acquisition characterization of renal masses [2]. If this can be realized, radiation doses can be reduced significantly by eliminating the unenhanced CT acquisition [15]. The use of dual-energy CT iodine identification allows virtual noncontrast images in which the attenuation from iodine is subtracted from the contrast-enhanced image in an attempt to reproduce the baseline unenhanced attenuation. It is also possible to produce an "iodine overlay," in which the presence of iodine in the tissues is indicated by a superimposed color map [13, 38]. Both of these image sets have been investigated to determine their usefulness in allowing single $\mathrm{CT}$ acquisition for renal mass evaluation.

Virtual noncontrast images-Graser et al. [10] described the possible use of virtual noncontrast images to replace true unenhanced CT images by comparing the measured attenuation values on the true unenhanced images versus on the virtual noncontrast images. More recently the virtual noncontrast images were used as the baseline attenuation against which the enhanced images were compared to identify the presence of enhancement; this comparison showed good correlation with results comparing the true unenhanced images with the enhanced images [15]. One area of concern is in regard to the accuracy of the attenuation measurements of structures on the virtual noncontrast images versus those obtained from true unenhanced images. Recent quantitative comparative analysis of mean density measurements found no significant difference: The authors noted that "dual-energy CT-based virtual noncontrast images are a reasonable approximation of true unenhanced single-energy CT images" [39]. 
lodine-overlay images-The use of iodine-overlay images for the detection of actual iodine signal within the mass has also been studied. In this case, one would not rely on relative changes in attenuation to determine enhancement, but would determine enhancement by directly visualizing the presence of iodine within the center of a mass. Because renal cysts do not contain central blood flow, an iodine-overlay image should show a cyst as devoid of iodine signal (Fig. 4). In contrast, the vessels and tissues of a solid renal mass will accumulate iodine after contrast injection and the signal will be identified on the overlay image (Fig. 5). Brown et al. [13] first described this concept for use in renal mass evaluation based on dual-energy CT phantom studies. The clinical utility of iodine-overlay images alone for the diagnosis of a renal mass versus a renal cyst has since been reported (Hartman RP, et al., presented at the 2009 annual meeting of the RSNA).

This method may prove beneficial beyond the current practice of determining enhancement in a renal mass because it has potential to reduce the effect of renal cyst pseudoenhancement. Pseudoenhancement is an artifactual increase in the attenuation of a true renal cyst after contrast administration. Although the exact mechanism for pseudoenhancement is not completely understood, it can result in an erroneous increase in the attenuation of a renal cyst up to $20 \mathrm{HU}$ above the baseline attenuation on unenhanced $\mathrm{CT}$. The degree of pseudoenhancement can vary relative to cyst size, renal parenchymal enhancement, detector configuration, and scanning protocol [16, 17, 40, 41].

In contrast, an iodine-overlay image either shows signal in the mass or not. If signal is present, the mass is a solid lesion and further management, including excision, should be considered. If no iodine signal is present, the lesion is a cyst. In the future, monochromatic imaging, either synthesized from dual-energy CT or generated from photon-counting detector-based CT, could considerably minimize beam-hardening artifacts and provide abundant energy-dependent attenuation information that can be used to characterize renal masses.

As the virtual noncontrast images and iodine-overlay images are refined and studied, it is conceivable that one or both may be able to differentiate renal cysts from solid masses as well as or better than the current standard of practice. As mentioned, these images could be produced from a single contrast-enhanced $\mathrm{CT}$ acquisition and could eliminate dedicated unenhanced $\mathrm{CT}$ acquisition, thereby reducing examination time and radiation.

\section{Adrenal Adenoma}

Adrenal masses are identified in approximately 5\% of patients who undergo CT [42], with adrenal adenomas being the most common adrenal mass. Adrenal adenomas are characterized by a large amount of intracytoplasmic fat and typically appear hypoattenuated on unenhanced CT, measuring $10 \mathrm{HU}$ or less, and are referred to as lipid-rich adenomas.

The CT thresholds have been based on CT scans obtained at $120 \mathrm{kV}$. Boland et al. (presented at the 2008 annual meeting of the RSNA) reported that mean adenoma attenuation measurements at 80 and $120 \mathrm{kV}$ were $8.9 \mathrm{HU}$ (range, 18-33 HU) and $11.8 \mathrm{HU}$ (range, 18-38 HU) ( $p>0.05)$, respectively. Therefore, one should be cautious in evaluating adrenal masses using dual-energy CT until new thresholds are defined to reliably differentiate adenomas from nonadenomas [12]. 
Many adrenal masses are incidentally found on contrast-enhanced CT and often require delayed CT to calculate relative washout characteristics or additional imaging (MRI or CT) for further characterization. Virtual noncontrast images generated from contrast-enhanced dual-energy $\mathrm{CT}$ is a promising technique to provide information similar to true unenhanced CT [15] (Fig. 6). However, validation of the clinical utility of virtual noncontrast images in differentiating adenomas from nonadenomas remains necessary.

Up to $30 \%$ of adrenal adenomas do not contain a large amount of lipids. Therefore, characterization with unenhanced CT followed by contrast-enhanced and delayed CT is frequently required. Of note, $\mathrm{CT}$ scans obtained at the same parameters in each phase of unenhanced, contrast-enhanced, and delayed dual-energy $\mathrm{CT}$ acquisition should be used to measure $\mathrm{CT}$ attenuation values to define contrast washout characteristics.

\section{Urinary Stone in lodinated Solution (Virtual Noncontrast Images)}

As previously noted, unenhanced CT is considered the reference standard for the detection of urinary stones [20,21]. On contrast-enhanced CT, high-attenuation urinary stones can be obscured by high-attenuation iodinated contrast material in the renal parenchyma or collecting systems. Routine abdominal CT usually does not include unenhanced CT; therefore, renal stones can be missed. On the other hand, unenhanced CT is routinely performed as part of CT urography for hematuria workup because the prevalence of stone disease is high in this patient population [43]. If virtual noncontrast scans reconstructed from contrast-enhanced CT allow detection of urinary stones, there is potential for routine abdominal $\mathrm{CT}$ to increase the detection rate of unsuspected stones or eliminate true unenhanced CT from the CT urography protocol (Fig. 7).

In a phantom study, more than $95 \%$ of 2- to 4-mm stones immersed in solution with diluted iodinated contrast material were detectable using the virtual noncontrast technique except in extremely highly concentrated iodine solution. Iodine subtraction failed when the attenuation value of the iodine solution reached a maximal $\mathrm{CT}$ value $(3,070 \mathrm{HU})$ on low-kV images [44]. In a clinical study, virtual noncontrast images created from nephrographic phase CT images allowed detection of urinary stones with a sensitivity of $74.3 \%$ (26/35) on a per-stone basis [45]. However, the virtual noncontrast images did not improve the stone detection rate compared with the original nephrographic phase CT. In another clinical study, virtual noncontrast images created from urographic phase CT images of CT urography were evaluated. Virtual noncontrast images allowed detection of only 53\% of stones, although specificity was $100 \%$. The poor detection rate was mainly due to its inability to detect 1 - to 2-mm stones (Takahashi N, et al., presented at the 2008 annual meeting of the Society of Uroradiology, Abdominal Radiology Course).

\section{Urothelial Tumor Detection}

Detection of urothelial tumor relies on contrast between the tumor and surrounding urine. During the parenchymal phase of contrast enhancement, urothelial tumor shows increased enhancement while urine remains unenhanced [46]. During the urographic phase, tumor becomes deenhanced, whereas urine shows increased enhancement. The iodine enhancement technique may be useful to improve tumor conspicuity particularly when only a small 
amount of iodine contrast material can be used because of renal insufficiency or when only a slow rate of IV injection of iodine contrast material is allowed. In a phantom study simulating the urographic phase, the tumor-to-urine contrast-to-noise ratio increased 35\% and lesion conspicuity improved in $67 \%$ of lesions using a nonlinear sigmoidal blending technique when the iodine concentration of urine phantom was $250 \mathrm{HU}$. Lesion conspicuity did not improve when the iodine concentration of urine phantom was above $500 \mathrm{HU}$ because the tumor-to-urine contrast was already high (Takahashi N, et al., presented at the 2008 annual meeting of the RSNA).

\section{Current Issues Related to Dual-Energy CT}

There are several limitations regarding dual-energy CT scanning including equipment expense, increased images generated per study, technical learning curves, and lack of reimbursement. Regarding equipment expense, dual-energy CT scanners are more expensive than conventional MDCT scanners. When a dual-energy CT scanner is purchased and installed, resources are frequently directed to expansion and improvements for cardiac CT and therefore access to dual-energy CT for noncardiac (e.g., genitourinary) applications could be limited.

An additional challenge for dual-energy $\mathrm{CT}$ is the number of images generated per case with dual-energy CT, which is considerably greater when compared with conventional MDCT. For example, a single dual-energy CT examination might include sets of low, high, and blended anatomic images as well as additional reconstructed images for dual-energy postprocessing. Further research and progress will need to address optimal workflow for managing and archiving the huge data sets from dual-energy CT.

Additional learning curves are required for CT technologists and radiologists because dualenergy CT is more labor intense and time consuming than conventional MDCT. CT technologists need to spend more time and effort to scan, reconstruct, and process dualenergy CT image acquisitions. Radiologists also require additional training to accurately interpret dual-energy CT scans. Working collaboratively, a team of CT technologists, radiologists, and medical physicists is best capable of building optimal dual-energy CT protocols. Although additional and new information is provided to the radiologist from images generated by dual-energy CT, such as the virtual noncontrast images, charges can be made for only contrast-enhanced CT. There are currently no reimbursement current procedural terminology (CPT) codes for dual-energy CT postprocessing or interpretation.

\section{Future Directions}

Continued optimization of the techniques described in this article will provide more accurate detection and characterization of genitourinary disease. CT urography using dual-energy CT technology could become a compressive genitourinary imaging study with a potential reduction of radiation exposure to the patient. With optimized spectra of the low- and highenergy x-ray beams, stone characterization and iodine quantification will become more accurate and precise. Optimization of the spectra usually involves a combined consideration among spectra separation, noise, and radiation dose. Added filtration in the high-energy 
beam represents one of the recent advances in optimizing the spectra [8], but additional research is required to further improve dual-energy CT performance.

Noise in virtual noncontrast images is a big concern because dual-energy CT processing usually involves a magnification of image noise, the magnitude of which is highly dependent on the spectra separation [47]. Currently, a significant amount of smoothing is usually applied to reduce the noise in virtual noncontrast images, which may degrade the image quality. By optimizing the spectra of the low- and high-energy x-ray beams, the quality in virtual noncontrast images will also be improved.

Monochromatic imaging, which is enabled by projection-domain dual-energy processing, would allow a significant reduction of beam-hardening artifacts [48]. It is expected that monochromatic imaging will improve the $\mathrm{CT}$ number accuracy and provide more quantitative information of the structure. In the future, patients may be scanned in the dualenergy mode by default because it will add more diagnostic information to what can be achieved by the single-energy mode especially if there is no penalty of additional radiation dose by using the dual-energy mode when patient size is not too big [19, 49].

Finally, photon-counting detectors are under active investigation [50, 51]. These detectors have several advantages over conventional energy-integrated detectors including optimal energy weighting, more flexible selection of energy bins, and better spectra separation. Photon-counting detector-based CT technology is expected to provide even more accurate material-specific information and more dose-efficient clinical applications for dual-energy CT.

\section{Conclusions}

Dual-energy CT will play an expanded role in the evaluation of patients with genitourinary disease in the future by providing expanded diagnostic information from CT images. Material characterization and diagnostic accuracy will be improved using dual-energy CT without a penalty from increased radiation dose requirements. Additional research and workflow enhancements will provide the necessary steps toward determination of the optimal role of dual-energy CT in the future.

\section{Acknowledgments}

J. G. Fletcher receives grant support from Siemens Medical Solutions.

\section{References}

1. Fletcher JG, Takahashi N, Hartman R, et al. Dual-energy and dual-source CT: is there a role in the abdomen and pelvis? Radiol Clin North Am. 2009; 45:41-57. [PubMed: 19195533]

2. Flohr TG, McCollough CH, Bruder H, et al. First performance evaluation of a dual-source CT (DSCT) system. Eur Radiol. 2006; 16:256-268. [PubMed: 16341833]

3. Holmes DR 3rd, Fletcher JG, Apel A, et al. Evaluation of non-linear blending in dual-energy computed tomography. Eur J Radiol. 2008; 68:409-413. [PubMed: 18990521]

4. Primak AN, Fletcher JG, Vrtiska TJ, et al. Noninvasive differentiation of uric acid versus non-uric acid kidney stones using dual-energy CT. Acad Radiol. 2007; 14:1441-1447. [PubMed: 18035274] 
5. Stolzmann P, Kozomara M, Chuck N, et al. In vivo identification of uric acid stones with dualenergy CT: diagnostic performance evaluation in patients. Abdom Imaging. 2009 Sep 2. Epub ahead of print.

6. Stolzmann P, Leschka S, Scheffel H, et al. Characterization of urinary stones with dual-energy CT: improved differentiation using a tin filter. Invest Radiol. 2010; 45:1-6. [PubMed: 19996763]

7. Chandarana H, Godoy MC, Vlahos I, et al. Abdominal aorta: evaluation with dual-source dualenergy multidetector CT after endovascular repair of aneurysms-initial observations. Radiology. 2008; 249:692-700. [PubMed: 18812561]

8. Primak AN, Ramirez Giraldo JC, Liu X, Yu L, McCollough CH. Improved dual-energy material discrimination for dual-source CT by means of additional spectral filtration. Med Phys. 2009; 36:1359-1369. [PubMed: 19472643]

9. Carmi R, Naveh GA, Altman A. Material separation with dual-layer CT. IEEE. 2005; M03M367:1876-1878.

10. Graser A, Johnson TR, Chandarana H, Macari M. Dual energy CT: preliminary observations and potential clinical applications in the abdomen. Eur Radiol. 2009; 19:13-23. [PubMed: 18677487]

11. Macari M, Spieler B, Kim D, et al. Dual-source dual-energy MDCT of pancreatic adenocarcinoma: initial observations with data generated at $80 \mathrm{kVp}$ and at simulated weighted-average $120 \mathrm{kVp}$. AJR. 2010; 194:144. [web]W27-W32.

12. Yeh BM, Shepherd JA, Wang ZJ, Teh HS, Hartman RP, Prevrhal S. Dual-energy and low-kVp CT in the abdomen. AJR. 2009; 193:47-54. [PubMed: 19542394]

13. Brown CL, Hartman RP, Dzyubak OP, et al. Dual-energy CT iodine overlay technique for characterization of renal masses as cyst or solid: a phantom feasibility study. Eur Radiol. 2009; 19:1289-1295. [PubMed: 19153744]

14. Schlomka JP, Roessl E, Dorscheid R, et al. Experimental feasibility of multi-energy photoncounting K-edge imaging in pre-clinical computed tomography. Phys Med Biol. 2008; 53:40314047. [PubMed: 18612175]

15. Graser A, Johnson TR, Hecht EM, et al. Dual-energy CT in patients suspected of having renal masses: can virtual nonenhanced images replace true nonenhanced images? Radiology. 2009; 252:433-440. [PubMed: 19487466]

16. Birnbaum BA, Hindman N, Lee J, Babb JS. Multidetector row CT attenuation measurements: assessment of intra- and interscanner variability with an anthropomorphic body CT phantom. Radiology. 2007; 242:109-119. [PubMed: 17185663]

17. Birnbaum BA, Hindman N, Lee J, Babb JS. Renal cyst pseudoenhancement: influence of multidetector CT reconstruction algorithm and scanner type in phantom model. Radiology. 2007; 244:767-775. [PubMed: 17709828]

18. Levi C, Gray JE, McCullough EC, Hattery RR. The unreliability of CT numbers as absolute values. AJR. 1982; 139:443-447. [PubMed: 6981306]

19. Yu L, Primak AN, Liu X, McCollough CH. Image quality optimization and evaluation of linearly mixed images in dual-source, dual-energy CT. Med Phys. 2009; 36:1019-1024. [PubMed: 19378762]

20. Smith RC, Rosenfield AT, Choe KA, et al. Acute flank pain: comparison of non-contrast-enhanced CT and intravenous urography. Radiology. 1995; 194:789-794. [PubMed: 7862980]

21. Smith RC, Verga M, Dalrymple N, McCarthy S, Rosenfield AT. Acute ureteral obstruction: value of secondary signs of helical unenhanced CT. AJR. 1996; 167:1109-1113. [PubMed: 8911160]

22. Fielding JR, Steele G, Fox LA, Heller H, Loughlin KR. Spiral computerized tomography in the evaluation of acute flank pain: a replacement for excretory urography. J Urol. 1997; 157:20712073. [PubMed: 9146582]

23. Chen MYM, Zagoria RJ. Can unenhanced helical computed tomography replace excretory urography for evaluation of patients with acute urinary tract colic? J Emerg Med. 1999; 17:299_ 303. [PubMed: 10195491]

24. Niall O, Russell J, MacGregor R, Duncan H, Mullins J. A comparison of noncontrast computerized tomography with excretory urography in the assessment of acute flank pain. J Urol. 1999; 161:534-537. [PubMed: 9915442] 
25. Yilmaz S, Sindel T, Arslan G, et al. Renal colic: comparison of spiral CT, US and IVU in the detection of ureteral calculi. Eur Radiol. 1998; 8:212-217. [PubMed: 9477267]

26. Hillman BJ, Drach GW, Tracey P, Gaines JA. Computed tomographic analysis of renal calculi.

27. Mostafavi MR, Ernst RD, Saltzman B. Accurate determination of chemical composition of urinary calculi by spiral computerized tomography. J Urol. 1998; 159:673-675. [PubMed: 9474123]

28. Mitcheson HD, Azmenhof RG, Bankoff MS, Prien EL. Determination of chemical composition of urinary calculi by computerized tomography. J Urol. 1983; 130:814-819. [PubMed: 6887427]

29. Newhouse JH, Prien EL, Amis ESJ, Dretler SP, Pfister RC. Computed tomographic analysis of urinary calculi. AJR. 1984; 142:545-548. [PubMed: 6607644]

30. Demirel A, Suma S. The efficacy of non-contrast helical computed tomography in the prediction of urinary stone composition in vivo. J Int Med Res. 2003; 31:1-5. [PubMed: 12635527]

31. Stolzmann P, Scheffel H, Rentsch K, et al. Dual-energy computed tomography for the differentiation of uric acid stones: ex vivo performance evaluation. Urol Res. 2008; 36:133-138. [PubMed: 18545993]

32. Graser A, Johnson TR, Bader M, et al. Dual energy CT characterization of urinary calculi: initial in vitro and clinical experience. Invest Radiol. 2008; 43:112-119. [PubMed: 18197063]

33. Matlaga BR, Kawamoto S, Fishman E. Dual source computed tomography: a novel technique to determine stone composition. Urology. 2008; 72:1164-1168. [PubMed: 18619656]

34. Boll DT, Patil NA, Paulson EK, et al. Renal stone assessment with dual-energy multidetector CT and advanced postprocessing techniques: improved characterization of renal stone compositionpilot study. Radiology. 2009; 250:813-820. [PubMed: 19244048]

35. Thomas C, Patschan O, Ketelsen D, et al. Dual-energy CT for the characterization of urinary calculi: in vitro and in vivo evaluation of a low-dose scanning protocol. Eur Radiol. 2009; 19:1553-1559. [PubMed: 19205704]

36. Israel GM, Bosniak MA. How I do it: evaluating renal masses. Radiology. 2005; 236:441-450. [PubMed: 16040900]

37. Siegel CL, Fisher AJ, Bennett HF. Interobserver variability in determining enhancement of renal masses on helical CT. AJR. 1999; 172:1207-1212. [PubMed: 10227490]

38. Johnson TR, Krauss B, Sedlmair M, et al. Material differentiation by dual energy CT: initial experience. Eur Radiol. 2007; 17:1510-1517. [PubMed: 17151859]

39. Sommer WH, Graser A, Becker CR, et al. Image quality of virtual noncontrast images derived from dual-energy CT angiography after endovascular aneurysm repair. J Vasc Interv Radiol. 2010; 21:315-321. [PubMed: 20097097]

40. Abdulla C, Kalra MK, Saini S, et al. Pseudoenhancement of simulated renal cysts in a phantom using different multidetector CT scanners. AJR. 2002; 179:1473-1476. [PubMed: 12438037]

41. Wang ZJ, Coakley FV, Fu Y, et al. Renal cyst pseudoenhancement at multidetector CT: what are the effects of number of detectors and peak tube voltage? Radiology. 2008; 248:910-916. [PubMed: 18632527]

42. Song JH, Chaudhry FS, Mayo-Smith WW. The incidental adrenal mass on CT: prevalence of adrenal disease in 1,049 consecutive adrenal masses in patients with no known malignancy. AJR. 2008; 190:1163-1168. [PubMed: 18430826]

43. Kawashima A, Glockner JF, King BF Jr. CT urography and MR urography. Radiol Clin North Am. 2003; 41:945-961. [PubMed: 14521203]

44. Takahashi N, Hartman RP, Vrtiska TJ, et al. Dual-energy CT iodine-subtraction virtual unenhanced technique to detect urinary stones in an iodine-filled collecting system: a phantom study. AJR. 2008; 190:1169-1173. [PubMed: 18430827]

45. Scheffel H, Stolzmann P, Frauenfelder T, et al. Dual-energy contrast-enhanced computed tomography for the detection of urinary stone disease. Invest Radiol. 2007; 42:823-829. [PubMed: 18007154]

46. Kim JK, Park SY, Ahn HJ, Kim CS, Cho KS. Bladder cancer: analysis of multi-detector row helical CT enhancement pattern and accuracy in tumor detection and perivesical staging. Radiology. 2004; 231:725-731. [PubMed: 15118111] 
47. Kelcz F, Joseph PM, Hilal SK. Noise considerations in dual energy CT scanning. Med Phys. 1979; 6:418-425. [PubMed: 492076]

48. Alvarez RE, Macovski A. Energy-selective reconstructions in X-ray computerized tomography. Phys Med Biol. 1976; 21:733-744. [PubMed: 967922]

49. Alvarez RE, Seppi E. A comparison of noise and dose in conventional and energy selective computed tomography. IEEE Trans Nucl Sci. 1979; 26:2853-2856.

50. Shikhaliev PM. Energy-resolved computed tomography: first experimental results. Phys Med Biol. 2008; 53:5595-5613. [PubMed: 18799830]

51. Boll DT, Patil NA, Paulson EK, et al. Focal cystic high-attenuation lesions: characterization in renal phantom by using photon-counting spectral CT-improved differentiation of lesion composition. Radiology. 2010; 254:270-276. [PubMed: 20032158] 

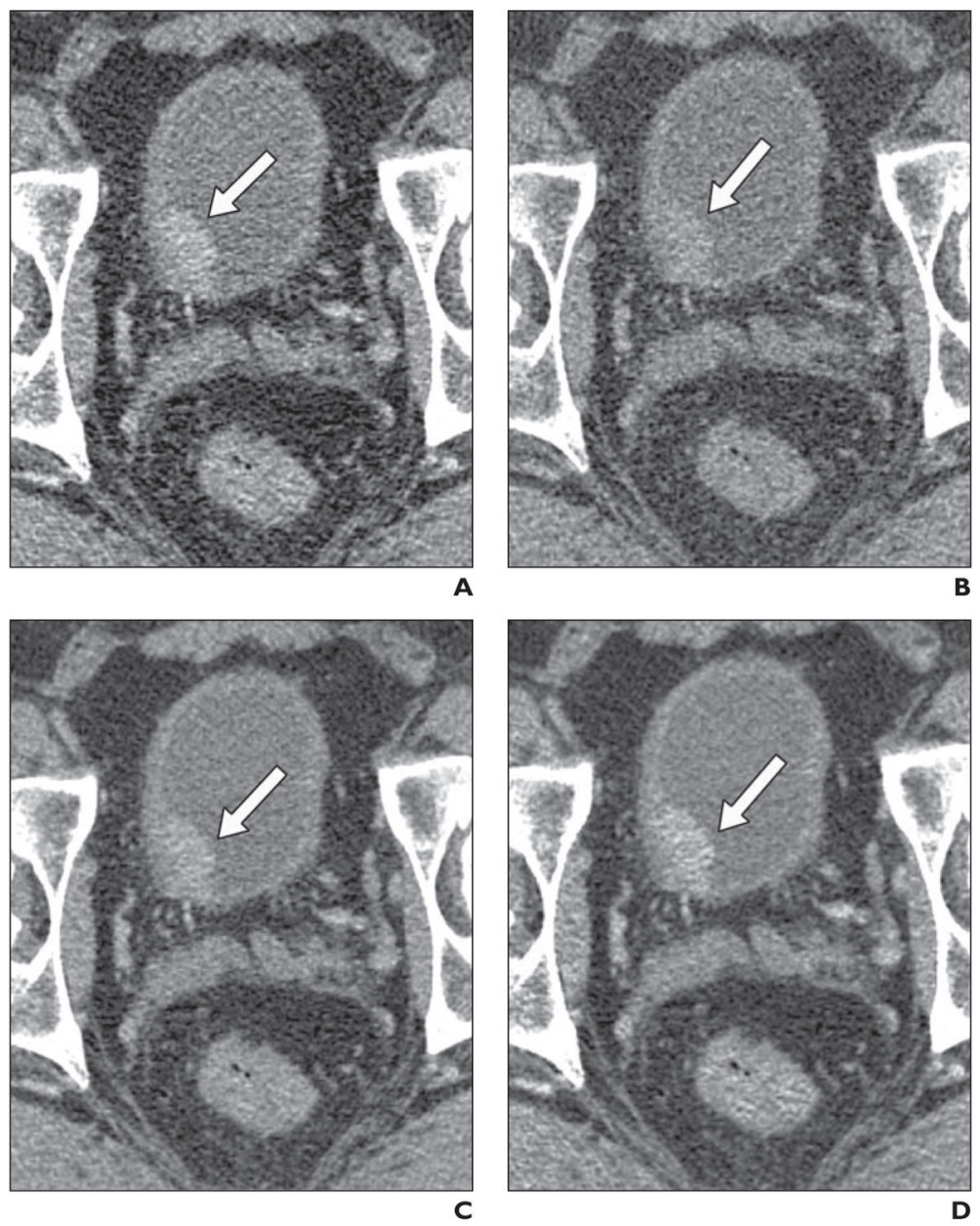

Fig. 1. Bladder cancer (arrow) in 51-year-old man

$\mathbf{A}$ and B, Axial CT images were obtained using dual-energy CT (100 kVp, A; $140 \mathrm{kVp}$ with tin filter, B). Note high bladder tumor-to-urine contrast and high image noise on lower-kV image (A) compared with higher-kV image (B).

C, Linear blended image (50\% from lower-kV image and 50\% from higher-kV image). Note tumor-to-urine contrast is between $\mathbf{A}$ and $\mathbf{B}$. Improvement of noise level is due to summation of two images.

D, Sigmoidal blending with center of $50 \mathrm{HU}$ and width of $20 \mathrm{HU}$. Note improvement of tumor-to-urine contrast compared with $\mathbf{C}$ while noise level in bladder lumen is maintained. This difference in contrast is because most signals of bladder tumor come from lower-kV image and most signals of bladder lumen come from higher-kV image. 


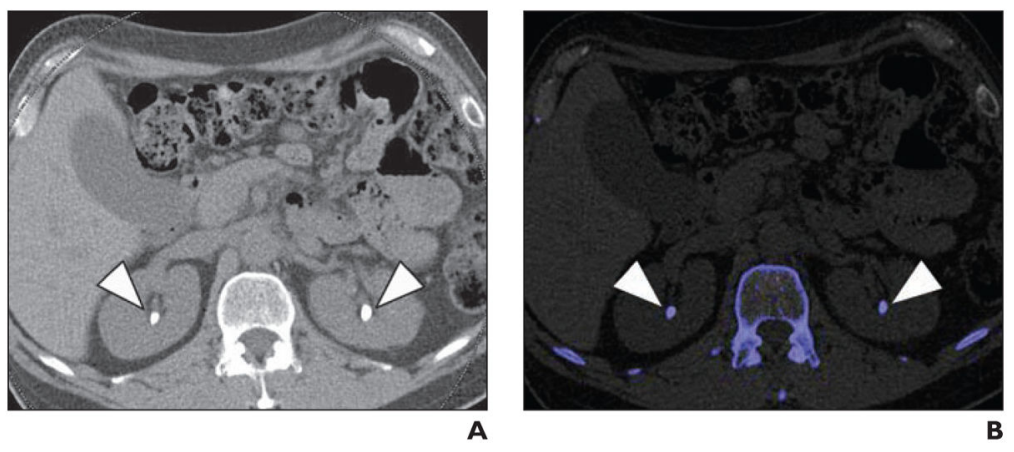

Fig. 2. Calcium oxalate stones (arrowheads) in 46-year-old man with history of gastric bypass, hyperoxaluria, and recurrent stones

A, Axial unenhanced image of mid kidneys show bilateral renal calculi.

B, Axial unenhanced image of mid kidneys show bilateral renal calculi (blue) color coded to represent non-uric acid stones. 

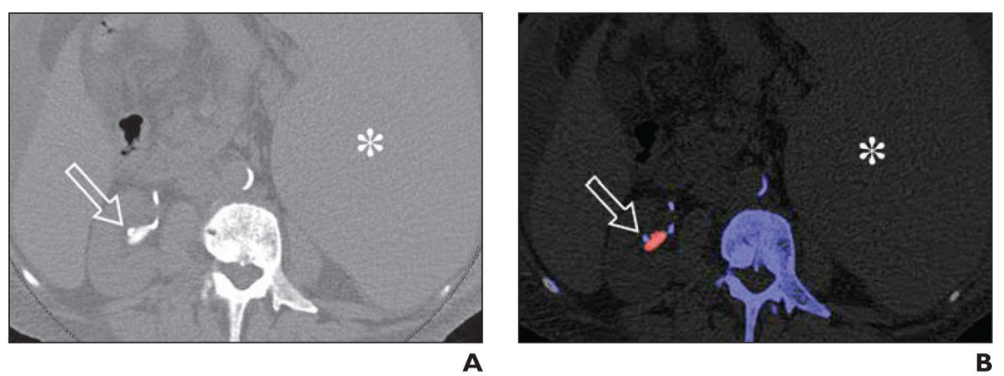

Fig. 3. Uric acid stones (arrow) in 54-year-old man with history of myelofibrosis and splenomegaly

A, Axial unenhanced image of mid right kidney shows right renal pelvic stone and adjacent catheter tubing.

B, Axial unenhanced image of mid right kidney shows color-coded stone in mid right kidney. Red color coding is most consistent with uric acid composition. Note adjacent colorcoded ureteral stent. Spleen (asterisk, A and B) is also enlarged. 

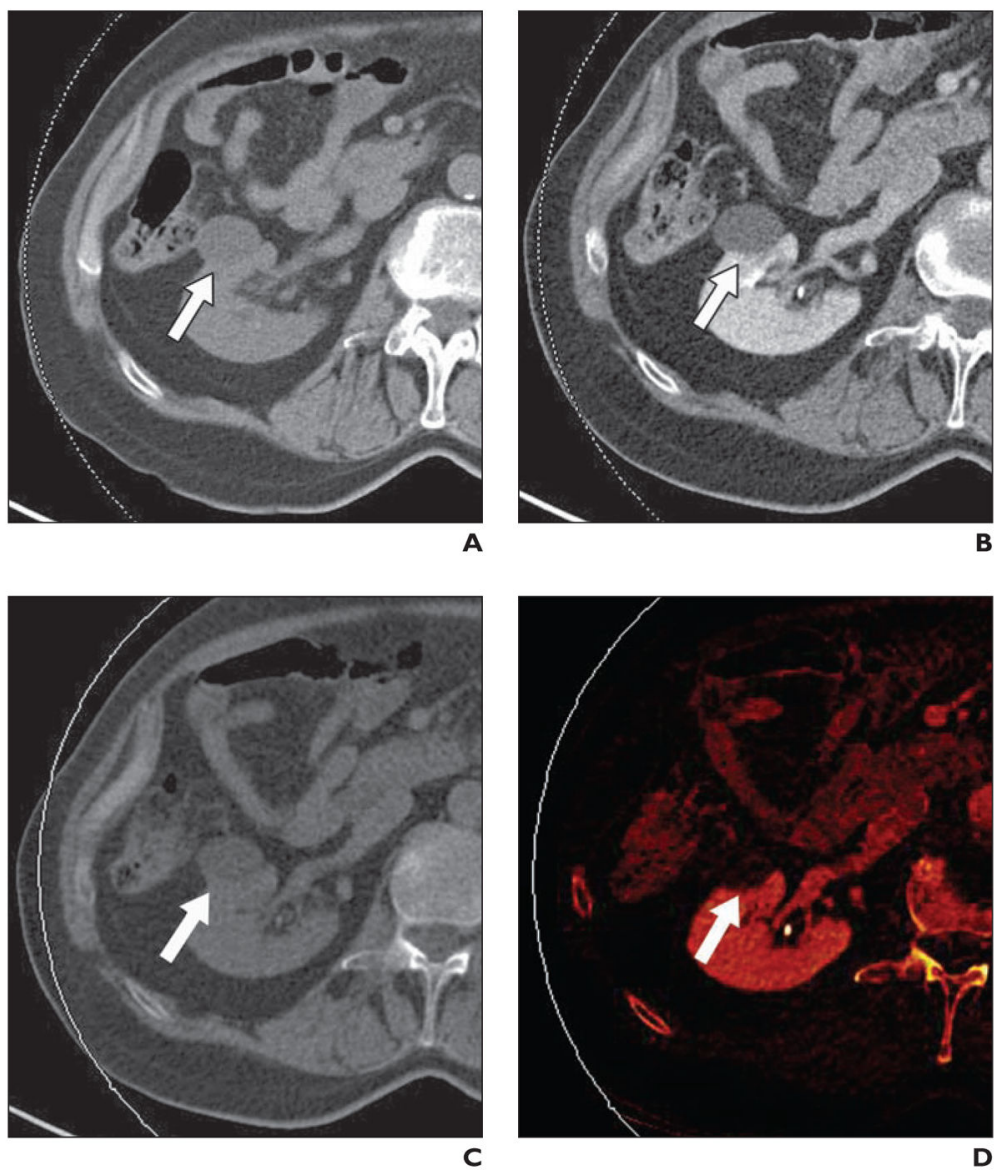

Fig. 4. Benign simple renal cyst (arrow) in 79-year-old man

A, Axial unenhanced image through low-attenuation mass in right kidney. Attenuation of lesion measures $7 \mathrm{HU}$.

B, Axial enhanced image through lesion shows no visual evidence of enhancement. Attenuation of lesion measures $8 \mathrm{HU}$. Absence of enhancement is diagnostic of benign renal cyst.

C, Axial virtual noncontrast image. Measured attenuation was nearly identical to true unenhanced value (6.2 HU).

D, Axial iodine-overlay image through lesion shows absence of central iodine signal; this finding is consistent with diagnosis of renal cyst. 

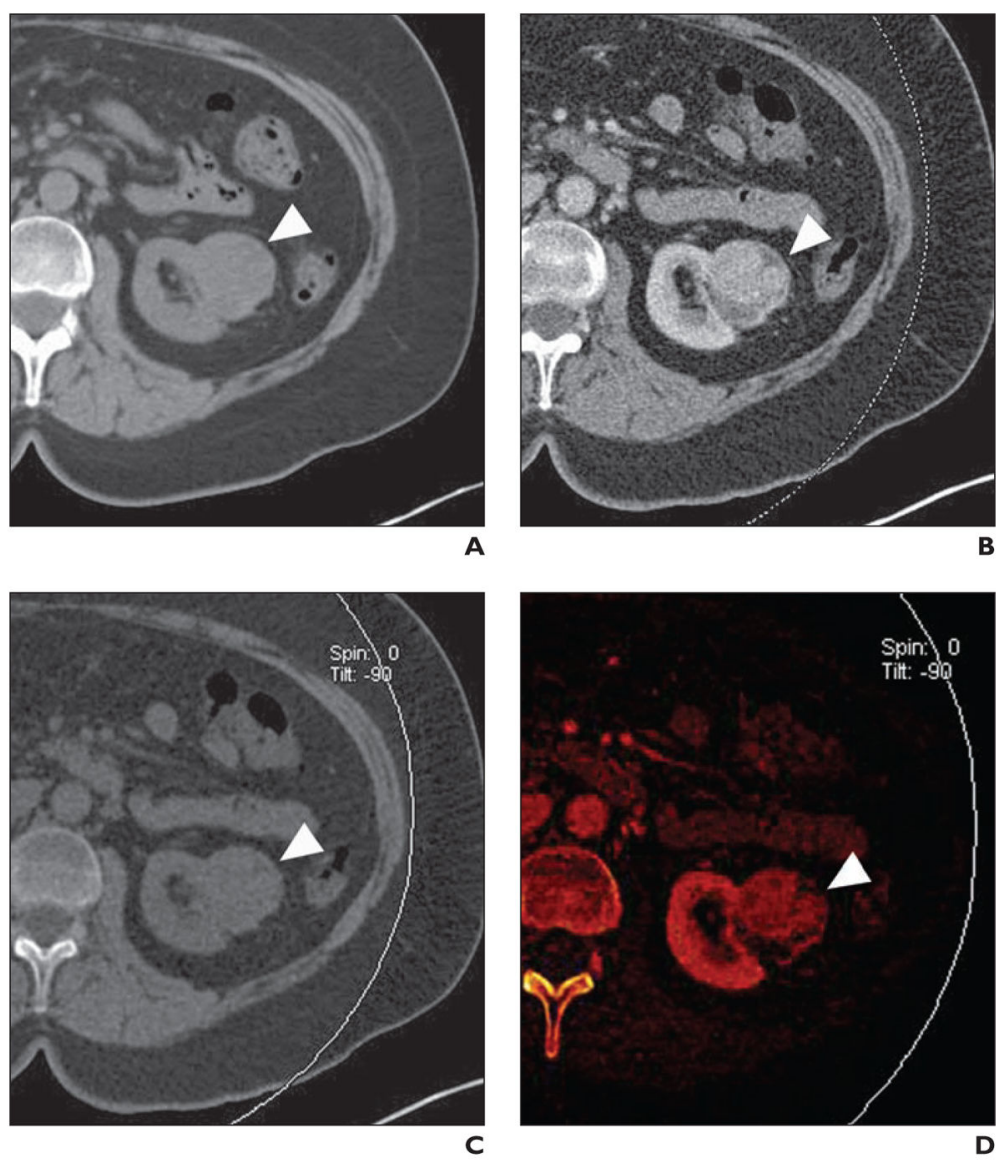

Fig. 5. Grade 3 clear cell renal cell carcinoma (RCC) (arrowhead) in 55-year-old woman A, Axial unenhanced image through mass in lower left kidney. Attenuation of lesion measures $26 \mathrm{HU}$.

B, Axial contrast-enhanced image through lesion shows heterogeneous enhancement. Attenuation of lesion measures $102 \mathrm{HU}$. Increased attenuation (> $20 \mathrm{HU}$ ) is diagnostic of solid renal mass. At surgery, mass was shown to be grade 3 clear cell RCC (highest possible grade $=$ grade 4 ).

C, Axial virtual noncontrast image generated from contrast-enhanced CT images.

D, Axial iodine-overlay image through lesion shows central iodine signal; this finding is consistent with diagnosis of solid renal mass. 

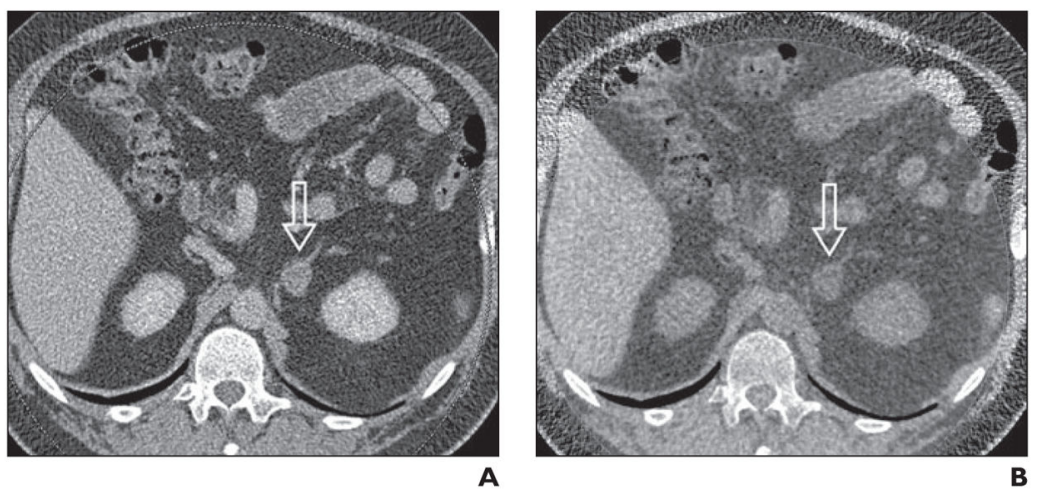

Fig. 6. Adrenal nodule (arrow) in 57-year-old man

A, Axial contrast-enhanced image through nodule in left adrenal gland. Attenuation of lesion measures $48 \mathrm{HU}$.

B, Virtual noncontrast CT image through left adrenal mass created from contrast-enhanced images lesion shows -4 HU. Low attenuation changes of mass are consistent with benign adenoma; however, further research and clinical validation are required. 

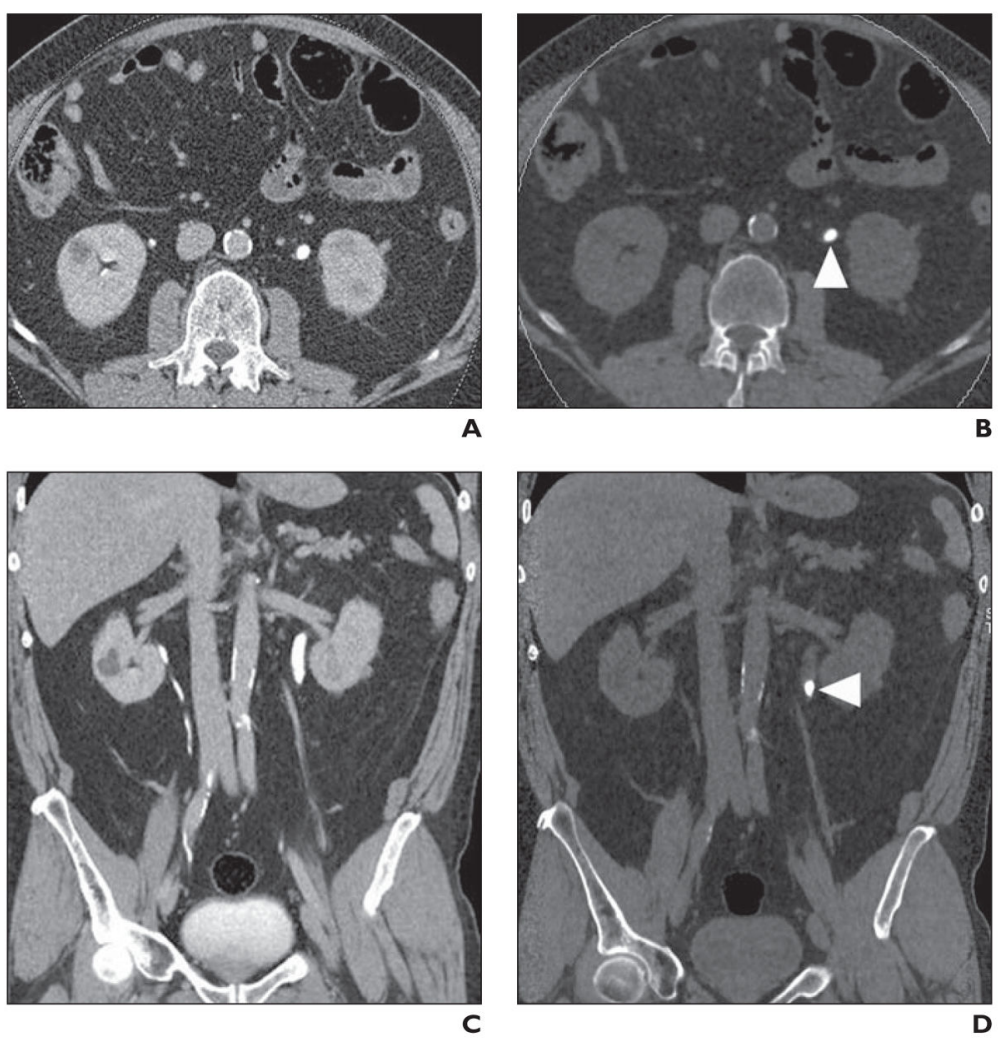

Fig. 7. Left ureteropelvic stone (arrowhead) in 65-year-old man visualized on virtual noncontrast image created from pyelographic phase

A, Axial urographic phase image through mid kidneys with contrast material within both ureters.

B, Axial virtual noncontrast image shows left ureteropelvic stone.

C, Coronal urographic phase image through urinary tract with opacification of segments of collecting systems of both kidneys.

D, Coronal virtual noncontrast image shows calculus in proximal left ureter. 


\section{TABLE 1}

Features of Dual-Energy CT Hardware Platforms

\begin{tabular}{l|l|l}
\hline Hardware Platform & Advantages & Disadvantages \\
\hline Dual-source CT & $\begin{array}{l}\text { Different beam filtration for each tube results in } \\
\text { wider spectral separation and improved material } \\
\text { discrimination }\end{array}$ & $\begin{array}{l}\text { No projection-domain dual-energy processing for helical } \\
\text { scans } \\
\text { Second tube has limited scanning field of view, requiring } \\
\text { cptimizes image quality } \\
\text { careful patient positioning for large patients }\end{array}$ \\
Rapid kV switching & $\begin{array}{l}\text { Allows dual-energy processing in projection space } \\
\text { Allows creation of monochromatic images, which } \\
\text { have the potential to reduce beam-hardening } \\
\text { artifacts }\end{array}$ & $\begin{array}{l}\text { Same beam filtration for both low- and high-energy } \\
\text { scanning, limited spectra separation } \\
\text { Difficult to adjust tube current for low- and high-energy } \\
\text { scans separately, usually leading to inferior image quality in } \\
\text { the low-energy scan } \\
\text { Potential for significant spectra overlap, which may } \\
\text { compromise performance of material characterization } \\
\text { Difficult to achieve similar image quality in low-energy data } \\
\text { as in high-energy data }\end{array}$ \\
\hline
\end{tabular}

